Contemporary Mathematics

Volume 17, 1998

\title{
Unstable Bundles in Quantum Field Theory
}

\author{
M. Asorey ${ }^{\dagger}$, F. Falceto ${ }^{\dagger}$ and G. Luzón ${ }^{\ddagger}$
}

\begin{abstract}
The relation between connections on 2-dimensional manifolds and holomorphic bundles provides a new perspective on the role of classical gauge fields in quantum field theory in two, three and four dimensions. In particular we show that there is a close relation between unstable bundles and monopoles, sphalerons and instantons. Some of these classical configurations emerge as nodes of quantum vacuum states in non-confining phases of quantum field theory which suggests a relevant role for those configurations in the mechanism of quark confinement in QCD.
\end{abstract}

\section{Introduction}

The role of principal bundles in the theory of gauge fields is now well established as the kinematical geometric background where classical and quantum fluctuations of gauge fields evolve. However, it is much less known that holomorphic bundles also play a fundamental role in the dynamics of the theory.

There are two cases where the theory of holomorphic bundles proved to be very useful in the analysis of the classical dynamics of Yang-Mills fields. The first application arises in the construction of self-dual solutions of four-dimensional Yang-Mills equations (instantons), i.e. connections which minimize the Yang-Mills functional in non-trivial bundles. $U(N)$ instantons in $S^{4}$ are in one-to-one correspondence with holomorphic bundles of rank $\mathrm{N}$ on $C P^{3}$ which are trivial along the canonical $U(1)$ fibres [29]. The second application occurs in 2-D Yang-Mills theory, where the Yang-Mills functional becomes an almost equivariantly perfect Morse function in the space of 2-D connections [8]. In particular, there are solutions of the classical Yang-Mills equations in every stratum of the corresponding moduli space of holomorphic bundles.

However, in quantum field theory all field configurations are necessary for the formulation of the theory. The quantum states in the Schrödinger representation are functionals on the space of classical gauge field configurations. The relevance of classical field configurations for the low energy dynamics of the theory can be

Based on the Lectures given by M. Asorey at the "Conference on Secondary Calculus and Cohomological Physics", Moscow, Aug. 24-31, 1997 and at the "Workshop on Algebraic Geometry and Physics", Medina del Campo, Sep. 16-20, 1997.

We acknowledge CICYT for partial financial support under grant AEN97-1680. 
derived from the existence of critical points of the modulus of vacuum functionals. In particular the existence of nodal configurations for the vacuum functional has interesting consequences on the degeneracy of the quantum vacuum and the existence of phase transitions.

In this paper we point out that a common feature of all classical configurations that appear as nodal points of quantum vacua of gauge theories is their relation with 2-dimensional unstable bundles. Holomorphic bundles also appear in string theory and F-theory, although we shall not discuss here these very interesting recent applications (see $[\mathbf{1 3}][\mathbf{1 4}]$ for details).

\section{Unstable bundles.}

Let $\Sigma$ be a compact Riemann surface and let us recall the definition of holomorphic vector bundles.

Definition 2.1. A holomorphic bundle is a vector bundle $E\left(\Sigma, \mathbb{C}^{N}\right)$ with holomorphic transition functions.

The transition functions of a holomorphic bundle $g_{i j}(x) \in G L(N, \mathbb{C})$ satisfy, besides the compatibility conditions

$$
\begin{array}{cl}
g_{i i}(x)=\mathbb{I}_{N} & \text { for } x \in U_{i} \\
g_{i j}(x) g_{j k}(x) g_{k i}(x)=\mathbb{I}_{N} & \text { for } x \in U_{i} \cap U_{j} \cap U_{k},
\end{array}
$$

the holomorphic condition

$$
\partial_{\bar{z}} g_{i j}(x)=0 \quad \text { for } x \in U_{i} \cap U_{j} .
$$

An hermitean structure $\mathfrak{h}$ can be defined on the same vector bundle $E$ by unitary transition functions, i.e. $u_{i j}(x)=h_{i}^{-1} g_{i j} h_{j} \in U(N)$.

Definition 2.2. Given an hermitean structure structure $\mathfrak{h}$ in $E\left(\Sigma, \mathbb{C}^{N}\right)$ a connection $D: \Gamma(E) \rightarrow \Lambda_{1} \otimes \Gamma(E)$ is said to be unitary or compatible with $\mathfrak{h}$ if

$$
d \mathfrak{h}\left(\sigma_{1}, \sigma_{2}\right)=\mathfrak{h}\left(D \sigma_{1}, \sigma_{2}\right)+\mathfrak{h}\left(\sigma_{1}, D \sigma_{2}\right)
$$

for any pair of local sections $\sigma_{1}, \sigma_{2}$ of $E$.

DEFINITION 2.3. For any given hermitean structure $\mathfrak{h}$ of the holomorphic bundle $E\left(\Sigma, \mathbb{C}^{N}\right)$ there exists a unique linear connection $D$ on $E$ compatible with $\mathfrak{h}$ which satisfies $D_{\bar{z}} \sigma=0$ for any local holomorphic section $\sigma$ of $E$. Such a connection is called the canonical connection of $E$ with respect to $\mathfrak{h}$.

In local coordinates $D_{\bar{z}}=\partial_{\bar{z}}+h^{-1} \partial_{\bar{z}} h$ where $h: U \rightarrow G L(N, \mathbb{C})$ are the coordinates of a given local holomorphic frame in a local unitary trivialization of $E$. Notice that the local expression does not depend on the choice of such a frame and only depends on the local trivialization of $E$ and the holomorphic structure $[\mathbf{1 7}]$.

Conversely, given a unitary connection $D$ on $E$ with respect to the hermitean structure $\mathfrak{h}$ there exists a unique holomorphic structure on $E$ whose canonical connection is $D$. This follows from the fact that the local sections $\sigma$ satisfying the condition $D_{\bar{z}} \sigma=0$ define a holomorphic bundle on $E$, and can be summarized in the following terms. 
Proposition 2.1. There is a one-to-one correspondence between the space of holomorphic vector bundles of rank $N$ over a compact Riemann surface $\Sigma$ and the space of $U(N)$ unitary connections $\mathcal{A}$ over $\Sigma$ with respect to a given hermitean structure $\mathfrak{h}$ of the corresponding vector bundle.

In four dimensions there is a similar correspondence, but in such a case the canonical connections associated to holomorphic bundles must be selfdual. In two dimensions there is no constraint on the associated unitary connections.

According to Mumford [24] there is a particular class of holomorphic vector bundles which is distinguished by its stability properties: the class of stable vector bundles.

Definition 2.4. Given a holomorphic bundle over $\Sigma$ the ratio $\mu(E)=c(E) / r(E)$ of its first Chern class $c(E)$ by its rank $r(E)$ is called the slope of $E$.

Definition 2.5. A holomorphic vector bundle $\mathrm{E}$ is stable if there is no holomorphic sub-bundle $F$ with higher or equal slope, i.e. $\mu(F)<\mu(E)$. If there is no sub-bundle $F$ with higher slope $(\mu(F) \leq \mu(E)), E$ is said to be semi-stable, and if there exists a sub-bundle $F$ with higher slope $(\mu(F)>\mu(E)), E$ is said to be unstable.

For genus $g>1$ the space of stable bundles is a dense subset of the space of holomorphic bundles and its moduli space $\mathfrak{m}_{\mathrm{s}}$ is a smooth manifold. These and other nice features of stable bundles focused the interest on their study. However, in quantum field theory unstable bundles are acquiring a prominent role in recent applications.

Let us consider some examples which appear in the physical applications

EXAMPLE 2.1. There is always a trivial example of holomorphic bundle. It corresponds to $E=\Sigma \times \mathbb{C}^{N}$ with constant sections as holomorphic sections. Any basis of $\mathbb{C}^{N}$ defines a global frame of holomorphic sections that does not require nontrivial transition functions. $E$ is a semi-stable vector bundle. The corresponding canonical connection associated to any hermitean structure is $A_{\bar{z}}=h^{-1} \partial_{z} h$ with $h: \Sigma \rightarrow G L(N, \mathbb{C})$ globally defined on $\Sigma$. This connection corresponds to a classical vacuum of Yang-Mills theory.

EXAMPLE 2.2. A non-trivial holomorphic bundle can be associated to the abelian magnetic monopole.

Let us consider the complex structure of $S^{2}$ and the coordinates induced from $\mathbb{R}^{2}$ by the stereographic projection. A rank two holomorphic bundle $E\left(S^{2}, \mathbb{C}^{2}\right)$ is given by the transition function

$$
g_{ \pm}=\left(\begin{array}{cc}
z & 0 \\
0 & 1 / z
\end{array}\right)
$$

defined on the overlap $U_{+} \cap U_{-}=\{z ; 1 / R<|z|<R\}$ of the two charts $U_{-}=$ $\{z|| z \mid<R\}, U_{+}=\{z|| z \mid>R\}$ of $S^{2}$ defined for any radius $R>1$. This vector bundle is unstable because it always admits a line subbundle $L \subset E$ with first Chern class $c(L)=1$.

However, with the same holomorphic bundle and hermitean structure $\mathfrak{h}$ we can consider two different unitary trivializations of $\mathrm{E}$. The first one is given by two different unitary trivializations over $U_{-}$and $U_{+}$connected by the unitary transition 
function

$$
\phi_{ \pm}=\left(\begin{array}{cc}
z /|z| & 0 \\
0 & |z| / z
\end{array}\right) .
$$

The corresponding canonical connection, known as Dirac abelian magnetic monopole, is given by

$$
A_{\bar{z}}^{-}=\phi_{-}^{-1} \partial_{\bar{z}} \phi_{-}=\frac{1 / 2}{1+|z|^{2}}\left(\begin{array}{cc}
z & 0 \\
0 & -z
\end{array}\right) \quad \text { for } \quad|z|<R
$$

with

and

$$
\phi_{-}=\left(\begin{array}{cc}
\left(1+|z|^{2}\right)^{\frac{1}{2}} & 0 \\
0 & \left(1+|z|^{2}\right)^{-\frac{1}{2}}
\end{array}\right)
$$

$$
A_{\bar{z}}^{+}=\phi_{+}^{-1} \partial_{\bar{z}} \phi_{+}=\frac{1 / 2}{1+|z|^{2}}\left(\begin{array}{cc}
1 / \bar{z} & 0 \\
0 & -1 / \bar{z}
\end{array}\right) \quad \text { for } \quad|z|>R
$$

with

$$
\phi_{+}=\left(\begin{array}{cc}
\left(1+|z|^{2}\right)^{\frac{1}{2}} /|z| & 0 \\
0 & |z|\left(1+|z|^{2}\right)^{-\frac{1}{2}}
\end{array}\right)
$$

in the two charts $U_{+}, U_{-}$of $S^{2}$. Since the unitary trivializations of $E$ were not globally defined the connection between its two holomorphic trivializations is defined modulo $\phi_{ \pm}$, i.e.

$$
\phi_{+}=g_{ \pm}^{-1} \phi_{-} \phi_{ \pm}
$$

The matrices $\phi_{+}$and $\phi_{-}$transform the unitary trivializations into holomorphic trivializations.

There is, however, a global unitary trivialization of $E$ where the canonical connection reads

$$
A_{\bar{z}}=\varphi_{-}^{-1} \partial_{\bar{z}} \varphi_{-}=\varphi_{+}^{-1} \partial_{\bar{z}} \varphi_{+}=\frac{1}{\left(1+|z|^{2}\right)^{2}}\left(\begin{array}{cc}
-z & -1 \\
z^{2} & z
\end{array}\right),
$$

with

$\varphi_{-}=\left(\begin{array}{cc}z & 1 \\ -\left(1+|z|^{2}\right)^{-1} & \bar{z}\left(1+|z|^{2}\right)^{-1}\end{array}\right) ; \varphi_{+}=\left(\begin{array}{cc}1 & 1 / z \\ -z\left(1+|z|^{2}\right)^{-1} & |z|^{2}\left(1+|z|^{2}\right)^{-1}\end{array}\right)$

Notice that in this case since the unitary trivialization is globally defined $\varphi_{-}=$ $g_{ \pm} \varphi_{+}$. It is obvious that in any of the formulations there is line sub-bundle with first Chern class $c(E)=1$. Thus, the bundle $E$ is unstable.

The group $\mathcal{G}^{\mathbb{C}}$ of base preserving linear automorphisms of $\mathrm{E}$ establishes an equivalence relation between different holomorphic structures. In particular the two holomorphic bundles of example 2.2 are equivalent. The unitary automorphism

$$
g_{-}=\frac{1}{\left(1+|z|^{2}\right)^{\frac{1}{2}}}\left(\begin{array}{cc}
-\bar{z} & 1 \\
-1 & -z
\end{array}\right) \quad g_{+}=\frac{1}{\left(1+|z|^{2}\right)^{\frac{1}{2}}}\left(\begin{array}{cc}
|z| & -\bar{z} \\
1 / \bar{z} & |z|
\end{array}\right)
$$

establishes the equivalence between the two associated canonical connections.

The classification of holomorphic bundles over the sphere $S^{2}$ was carried out by Grothendieck [16]. Holomorphic bundles over the torus $T^{2}$ were classified by Atiyah [7]. The generalization for arbitrary genus can be found in Ref. [8] and references therein.

The space of holomorphic vector bundles over a Riemann surface, $\mathcal{H}$, can be identified, by Proposition 2.1, with the space of unitary connections $\mathcal{A}$ once a hermitean structure $\mathfrak{h}$ of $E$ is fixed. However, in both spaces there are two very different 
actions of automorphism groups. In $\mathcal{H}$ there is a natural action of automorphisms $\mathcal{G}^{\mathbb{C}}$ of $E$ as a complex vector bundle, whereas in $\mathcal{A}$ only the subgroup of unitary automorphisms $\mathcal{G}$ with respect to $\mathfrak{h}$ have a proper action. The two quotient spaces have a very different structure, they are called moduli space $\mathfrak{m}$ and orbit space $\mathcal{M}$, respectively.

However, none of those quotient spaces is a smooth manifold because the isotopy group of the automorphism groups actions are not the same for all orbits. In the case of unitary connections there two alternatives to provide the orbit space $\mathcal{M}$ with an smooth structure: whether restrict $\mathcal{A}$ to the space of irreducible (generic) connections $\mathcal{A}^{*}$, or consider the whole space of all connections $\mathcal{A}$ and restrict the automorphism group $\mathcal{G}$ to the group of unitary automorphisms $\mathcal{G}_{0}$ of $E$ that leave the fiber over a given point of $\Sigma$ invariant (pointed automorphisms). In both cases the corresponding orbit spaces, $\mathcal{M}^{*}=\mathcal{A}^{*} / \mathcal{G}, \mathcal{M}_{0}=\mathcal{A} / \mathcal{G}_{0}$ are smooth manifolds. In the case of holomorphic bundles we have not such alternatives because the variety of isotopy groups is wider. The one-dimensional subgroup of trivial automorphisms of the form $\exp (i \alpha) \mathbb{I} \in \mathcal{G}^{\mathbb{C}}$ leaves any holomorphic bundle invariant. All isotopy groups contain this trivial subgroup. But the isotopy groups of stable, semi-stable and unstable vector bundles are quite different as the following results point out.

Proposition 2.2. The only automorphisms which leave a stable bundle invariant are the trivial automorphisms of the form $\exp (i \alpha) \mathbb{I}$.

See Ref. [26] for a proof.

Proposition 2.3. The only pointed automorphism which leave a semi-stable bundle invariant is the trivial automorphism $\mathbb{I}$.

Proof. Let $\Phi$ be a pointed automorphism leaving the holomorphic structure of $E$ invariant. $\Phi$ is, thus, a holomorphic section of vector bundle End $E$ of endomorphisms of $E$, i.e. $\phi \in H^{0}(M$, End $E)$. We can associate to $\Phi$ an holomorphic section of the adjoint bundle ad $E, \xi=\Phi-\mathbb{I}$. By construction $\xi$ vanishes $(\xi(x)=0)$ at the point $x \in \Sigma$ where $\Phi(x)=\mathbb{I}$. Then, the holomorphic line sub-bundle $L_{\xi}$ of ad $E$ induced by $\xi$ must have a positive Chern class $c\left(L_{\xi}\right)>0$. Now, since ad $E$ inherits the semi-stable character of $E$ (see. e.g. [8] for a proof) and has 0 slope, this is not possible unless $\xi \equiv 0$, i.e. $\Phi=\mathbb{I}$.

The results point out the differences between isotopy groups of the different types of holomorphic bundles over a Riemann surface. The isotopy group of stable bundles only contains trivial automorphisms, whereas that of semi-stable bundles might contain some non-trivial automorphisms but they can never leave invariant any fiber of the bundle. The following result shows that the isotopy group of unstable bundles can contain pointed automorphisms.

Proposition 2.4. Any unstable bundle $E$ defined over the sphere with $c(E)=$ 0 is invariant under some pointed automorphisms.

Proof. Since $E$ is unstable the adjoint bundle ad $E$ is also unstable. There exists, thus, a sub-bundle $F$ of $\operatorname{rank} M$ of ad $E$ with $c(F)>0$. The Riemann-Roch theorem establishes that

$$
H^{0}(\Sigma, F)-H^{1}(\Sigma, F)=c(F)+M .
$$

Since $c(F)>0$, the bundle $F$ has more holomorphic sections than its rank. This implies that there is a linear combination of them, $\phi \in H^{0}(\Sigma, F)$, which vanish at 
a given point. The automorphisms of $E$ generated by this holomorphic section $\phi$ leave the bundles $F$ and $E$ invariant.

It follows from the previous discussion that the moduli space $\mathfrak{m}_{\mathrm{s}}$ of equivalent stable bundles over a Riemann surface is a finite dimensional smooth manifold, whereas the orbit space $\mathcal{M}^{*}=\mathcal{A}^{*} / \mathcal{G}$ of all irreducible connections is an infinite dimensional manifold. The characterization of stable bundles by the irreducible (projective) unitary representation of the first homotopy group of $\Sigma$ due to Narasimhan and Seshadri [26], provides a very useful approach to analyze the geometrical structure of the corresponding moduli space $\mathfrak{m}_{\mathrm{s}}[\mathbf{2 8}][\mathbf{2 5}]$.

If we include semi-stable bundles the smooth nature of the quotient space disappears as they might have larger isotopy groups. There is, however, a way of associating a smooth manifold to the corresponding moduli $\mathfrak{m}_{\mathrm{ss}}$. The quotient space of all semi-stable bundles by the group of pointed automorphisms has a smooth structure [11]. The procedure can be extended to include classes of unstable bundles but it is not possible to find a manifold for the whole set of holomorphic bundles different from the space $\mathcal{H}$ itself because the dimension of the isotopy group of unstable bundles is unbounded above.

Let us illustrate these properties with the examples considered above.

In the case of Example 2.1, all the constant gauge transformations leave the trivial holomorphic bundle invariant, which is semi-stable. The unstable bundle of example 2.2 has four holomorphic sections $\chi_{(\mu)} ; \mu=0,1,2,3$ in the adjoint bundle. Indeed the sections

$$
\begin{array}{ll}
\chi_{(3)}^{-}=\chi_{(3)}^{+}=\left(\begin{array}{cc}
-1 & 0 \\
0 & 1
\end{array}\right) & \\
\chi_{(k)}^{-}=\frac{z^{k}}{1+|z|^{2}}\left(\begin{array}{cc}
0 & 1 \\
0 & 0
\end{array}\right) & k=0,1,2 \\
\chi_{(k)}^{+}=\frac{z^{k}}{1+|z|^{2}}\left(\begin{array}{cc}
0 & \bar{z} / z \\
0 & 0
\end{array}\right) & k=0,1,2
\end{array}
$$

satisfy that $D_{\bar{z}} \chi_{(\mu)}=0$ in the first trivialization. The section $\chi_{(2)}$ vanishes at $z=0, \chi_{(0)}$ at $z=\infty$ and $\chi_{(1)}$ at both points.

In the second trivialization these four holomorphic sections $\chi_{(\mu)} ; \mu=0,1,2,3$ of the adjoint bundle read

$$
\begin{aligned}
& \chi_{(3)}=\frac{1}{1+|z|^{2}}\left(\begin{array}{cc}
|z|^{2}-1 & 2 \bar{z} \\
2 z & 1-|z|^{2}
\end{array}\right) \\
& \chi_{(k)}=\frac{z^{k}}{\left(1+|z|^{2}\right)^{2}}\left(\begin{array}{cc}
\bar{z} & -\bar{z}^{2} \\
1 & -\bar{z}
\end{array}\right) \quad k=0,1,2,
\end{aligned}
$$

and again $\chi_{(2)}$ vanishes at $z=0, \chi_{(0)}$ at $z=\infty$ and $\chi_{(1)}$ at both points. Since the holomorphic and hermitean structures are the same, and the only difference comes from the choice of different unitary trivializations, the sections (2.3) are just the transformed of (2.2) under the (chiral) complex gauge transformation (2.1).

However, the complete characterization of unstable bundles is quite complex [8]. The space $\mathcal{H}$ has a stratified structure. Every holomorphic bundle has a canonical filtration by subbundles $0=E_{0} \subset E_{1} \subset E_{2} \subset \cdots \subset E_{r}=E$, such that the quotients $D_{i}=E_{i} / E_{i-1}$ are semi-stable with strictly decreasing slopes, $\mu\left(D_{1}\right)>$ 
$\mu\left(D_{2}\right)>\cdots>\mu\left(D_{r}\right)$. The different strata of $\mathcal{H}$ are defined by all the holomorphic bundles which have the same pairs of integers $\left(r\left(D_{i}\right)_{i}, c\left(D_{i}\right)\right) ; i=1, \cdots, r$ in their canonical filtrations. In all the strata there are totally decomposable bundles $E=$ $D_{1} \oplus D_{2} \oplus \cdots \oplus D_{r}$ which are invariant under non-trivial automorphisms of the form $U=\exp \left(i \alpha_{1}\right) \mathbb{I}_{1} \oplus \exp \left(i \alpha_{2}\right) \mathbb{I}_{2} \oplus \cdots \oplus \exp \left(i \alpha_{r}\right) \mathbb{I}_{r}$. But, the isotopy group of the different orbits of a given stratum is not necessarily the same. The codimension of the stratum associated to a canonical filtration $\left(r\left(D_{i}\right)_{i}, c\left(D_{i}\right)\right) ; i=1, \cdots, r$

$$
\sum_{i<j}\left[c\left(D_{i}\right) r\left(D_{j}\right)-c\left(D_{j}\right) r\left(D_{i}\right)-r\left(D_{i}\right) r\left(D_{j}\right)(1-g)\right]
$$

reflects the fact that the increase of the dimension of the isotopy group for higher strata is compensated by the increase of the codimension of the strata modulo the change of dimension of the strata.

\section{Quantum Fermions}

The first application of unstable bundles appears in the study of $1+1$ dimensional QCD. The effective action of massless Dirac fermions becomes divergent for connections that correspond to unstable bundles, because of the existence of zero modes for the Dirac operator $[\mathbf{2 0}]$.

Proposition 3.1. The determinant of the Dirac operator $\not_{E}$ vanishes for connections associated to unstable bundles $E$ in any stratum.

Proof:. Since $E$ is unstable there is a subbundle $F$ with $c(F)>0$. By index theorem the asymmetry in the number of zero modes of the Dirac operator $\not D$ over the bundle $F$ with positive and negative chirality is given by

$$
\operatorname{dim} \operatorname{Ker} \not D_{F}^{+}-\operatorname{dim} \operatorname{Ker} \not D_{F}^{-}=c(F)>0 .
$$

In particular this implies the existence of non-trivial zero modes of positive chirality. Since

$$
0<\operatorname{dim} \operatorname{Ker} \not D_{F}^{+}+\operatorname{dim} \operatorname{Ker} \not D_{F}^{-} \leq \operatorname{dim} \operatorname{Ker} \not_{E}^{+}+\operatorname{dim} \operatorname{Ker} \not D_{E}^{-}
$$

we conclude the existence of zero modes of the Dirac operator $\not_{E}$.

\section{Chern-Simons Theory}

The topological character of the theory implies that the hamiltonian is identically null $H \equiv 0$. There are, however, two very stringent constraints on physical states $\Psi(A)$ : i) they only depend on the $A_{\bar{z}}$ components of unitary connections $A$, i.e.

and ii) they must satisfy the Gauss law

$$
\frac{\delta}{\delta A_{z}} \Psi(A)=0,
$$

$$
D_{\bar{z}} \frac{\delta}{\delta A_{\bar{z}}} \Psi\left(A_{\bar{z}}\right)=\frac{k}{\pi} \partial_{z} A_{\bar{z}} \Psi\left(A_{\bar{z}}\right) .
$$

The geometrical meaning of this law becomes more explicit if we multiply both sides of the equation by an arbitrary function $\chi: \Sigma \rightarrow \mathfrak{s l}(N, \mathbb{C})$. Integrating by parts on the left hand side we obtain

$$
-\int_{\Sigma} \operatorname{Tr}\left(D_{\bar{z}} \chi\right) \frac{\delta}{\delta A_{\bar{z}}} \Psi\left(A_{\bar{z}}\right)=\frac{k}{\pi} \int_{\Sigma} \operatorname{Tr} \chi \partial_{z} A_{\bar{z}} \Psi\left(A_{\bar{z}}\right),
$$


which encodes the transformation law of the physical states under infinitesimal chiral complex gauge transformations. The integration of Gauss law, thus, defines the action of the group of chiral or complex gauge transformations $\mathcal{G}^{\mathbb{C}}$ on the physical states $\Psi\left(A_{\bar{z}}\right)$. This action of $h \in \mathcal{G}^{\mathbb{C}}$ on $A_{\bar{z}}$ is given by

$$
{ }^{h} A_{\bar{z}}=h A_{\bar{z}} h^{-1}+h \partial_{\bar{z}} h^{-1},
$$

and the isomorphism between $\mathcal{A}$ and $\mathcal{H}$ induces an action of $\mathcal{G}^{\mathbb{C}}$ on $\mathcal{A}$ which extends that of ordinary unitary gauge transformations in $\mathcal{G}$.

Theorem 4.1. The quantum states of Chern-Simons theory vanish for all connections associated to unstable bundles defined on the sphere or the torus.

Proof. The case of the sphere trivially follows from the fact that every holomorphic bundle on $S^{2}$ is decomposable as a sum of line bundles. For the torus a more elaborated argument is required. Let us first consider unstable bundles which split into two stable bundles,

$$
E=D_{+} \oplus D_{-}
$$

with Chern classes $c\left(D_{ \pm}\right)= \pm 1$. Due to the low values of the genus of $\Sigma$ and the Chern classes of $D_{ \pm}$both stable bundles do admit a canonical decomposition of the form

$$
D_{+}=\left(\begin{array}{ccccc}
L_{0}^{\mu_{1}} & X_{1}^{2} & X_{1}^{3} & \cdots & X_{1}^{r} \\
0 & L_{0}^{\mu_{2}} & X_{2}^{3} & \cdots & X_{2}^{r} \\
0 & 0 & L_{0}^{\mu_{3}} & \cdots & X_{2}^{r} \\
& \cdots & \cdots & \cdots & \\
0 & 0 & \cdots & \cdots & L_{1}^{\mu_{r}}
\end{array}\right) \quad D_{-}=\left(\begin{array}{ccccc}
L_{-1}^{\nu_{1}} & Y_{1}^{2} & Y_{1}^{3} & \cdots & Y_{1}^{s} \\
0 & L_{0}^{\nu_{2}} & Y_{2}^{3} & \cdots & Y_{2}^{s} \\
0 & 0 & L_{0}^{\nu_{3}} & \cdots & Y_{2}^{s} \\
\cdots & \cdots & \cdots & \cdots & \cdots \\
0 & 0 & \cdots & \cdots & L_{0}^{\nu_{s}}
\end{array}\right)
$$

where $L_{n}^{\mu}$ denotes a line bundle with Chern class $c\left(E_{n}\right)=n$ and holonomy $\mu$. It is trivial to see that generically (for holonomies in generic relative positions) the bundle $D_{+} \oplus D_{-}$do not admit extensions, i.e. $H^{1}\left(D_{+} \otimes D_{-}^{*}\right)=0$. Thus, the orbits of split bundles $E=D_{+} \oplus D_{-}$define a dense subset of the space of unstable bundles of the corresponding strata. Moreover, those orbits define a dense subset in the space of all unstable bundles. Now, if we saturate both sides of Gauss law (4.1) with the holomorphic section $\chi$ of $H^{0}(\operatorname{ad} E)$ defined by

$$
\chi=\left(\begin{array}{cc}
r \mathbb{I}_{1} & 0 \\
0 & -s \mathbb{I}_{2}
\end{array}\right),
$$

the left hand side vanishes because $\chi$ is holomorphic $D_{\bar{z}} \chi=0$ whereas the right hand side becomes proportional to

$$
\begin{aligned}
\int_{\Sigma} \operatorname{Tr} \chi \partial_{z} A_{\bar{z}} & =\int_{\Sigma} \operatorname{Tr}\left(\chi \partial_{z} A_{\bar{z}}-A_{z} D_{\bar{z}} \chi\right)=\int_{\Sigma} \operatorname{Tr} \chi F(A) \\
& =2 \pi\left(s c\left(D_{+}\right)-r c\left(D_{-}\right)\right)=2 \pi(r+s),
\end{aligned}
$$

which is a non vanishing factor. Thus, consistency of Gauss law requires the vanishing of any physical state $\Psi$ for those connections. By continuity we conclude that the nodal set of $\Psi$ include all connections in the adherence of the corresponding strata, which proves the theorem, i.e.

$$
\Psi\left(A_{\mathrm{uns}}\right)=0 .
$$


This vanishing result is in agreement with the limit of the explicit expressions of Chern-Simons states for connections associated to semi-stable bundles [15] [11].

\section{Topologically Massive Gauge Theory}

In this case the theory has propagating degrees of freedom and it is nontopological. The hamiltonian is given by

$$
\mathbb{H}=-\frac{g^{2}}{2}\left\|\frac{\delta}{\delta A}+\frac{i k}{4 \pi} * A\right\|^{2}+\frac{1}{2 g^{2}}\|F(A)\|^{2}
$$

and the physical states $\Psi(A)$ are constrained by Gauss law

$$
-i D_{A}^{*} \frac{\delta}{\delta A} \Psi(A)=\frac{k}{4 \pi} * d A \psi(A) .
$$

Proposition 5.1. The quantum states of topologically massive gauge theories vanish for all connections which are reducible to unitary connections with non-trivial Chern class.

Proof. Let $A$ be a connection which is reducible to a unitary connection $A_{0}$ with non-trivial Chern class $c\left(A_{0}\right) \neq 0$. Because of the hermitean structure of $E$ the connection $A$ can be split into two components $A=A_{0} \oplus A_{1}$ with non-trivial Chern classes, $c\left(A_{0}\right)=-c\left(A_{1}\right) \neq 0$. Reducible connections always have a nontrivial isotopy group under the action of the group of gauge transformations $\mathcal{G}$. In particular, the section of the adjoint bundle

$$
\chi=\left(\begin{array}{cc}
s c\left(A_{0}\right) \mathbb{I}_{0} & 0 \\
0 & r c\left(A_{1}\right) \mathbb{I}_{1}
\end{array}\right),
$$

generates a one-parametric subgroup of automorphisms of $E$ which leave $A$ invariant. This follows from the trivial identity $D_{A} \chi=0$. Consequently,

$$
\int d^{2} x \operatorname{Tr} \chi D_{A}^{*} \frac{\delta}{\delta A} \Psi(A)=0,
$$

for any functional $\Psi(A)$, which implies that

$$
\int_{\Sigma} \operatorname{Tr} \chi d A \Psi(A)=0
$$

for physical states, because of Gauss' law. This is impossible, since

$\int_{\Sigma} \operatorname{Tr} \chi d A=\int_{\Sigma} \operatorname{Tr} \chi\left(d A+D_{A} A\right)=2 \int_{\Sigma} \operatorname{Tr} \chi F(A)=4 \pi\left[s c\left(A_{0}\right)^{2}+r c\left(A_{1}\right)^{2}\right] \neq 0$, unless the physical state $\Psi$ vanishes for that connection, i.e. $\Psi(A)=0$.

Now, reducible connections do not exhaust all nodal configurations of physical states. The following topological arguments show that any physical state must have additional nodes on irreducible connections.

Proposition 5.2. Non-trivial solutions of Gauss law exist if and only if $k$ is an integer. In such a case these solutions are in one-to-one correspondence with sections of a line bundle $\mathcal{E}_{k}\left(\mathcal{M}^{*}, \mathbb{C}\right)$ over the orbit space of irreducible connections, with non-trivial first Chern class $c_{1}\left(\mathcal{E}_{k}\right) \in H^{2}\left(\mathcal{M}^{*}, \mathbb{Z}\right) \equiv \mathbb{Z}$ 
Proof. Gauss law has in this case a simple geometric interpretation in terms of the hermitean $U(1)$ connection $\widetilde{\alpha}_{k}$ defined over the space of irreducible connections $\mathcal{A}^{*}$ by the one-form

$$
\widetilde{\alpha}_{k}(\tau)=\frac{k}{4 \pi}(* A, \tau)+\frac{k}{2 \pi}\left(D_{A} G_{A} * F(A), \tau\right) \quad \forall \tau \in T_{A} \mathcal{A}
$$

with $G_{A}=\left(D_{A}^{*} D_{A}\right)^{-1}$. Actually, the quantum Gauss law condition (5.2) can be written as

$$
D_{A}^{*} \nabla_{\widetilde{\alpha}_{k}} \psi(A)=0
$$

with

$$
\nabla_{\widetilde{\alpha}_{k}}=\frac{\delta}{\delta A}+i \widetilde{\alpha}_{k}
$$

which means that the quantum states are covariantly constant along the gauge fibers with respect to the $U(1)$ connection $\widetilde{\alpha}_{k}$. The existence of non-trivial solutions of the quantum Gauss condition (5.3) is possible if and only if the connection $\widetilde{\alpha}_{k}$ is trivial along the orbits of the group of gauge transformations $\mathcal{G}$ (modulo its center) $[\mathbf{5}][\mathbf{1}]$. The curvature 2 -form of $\widetilde{\alpha}_{k}$

$$
\widetilde{\Omega}_{k}(\widetilde{\tau}, \widetilde{\eta})=-\frac{k}{4 \pi}(\widetilde{\tau}, * \widetilde{\eta})+\frac{k}{2 \pi}\left(G_{A} *[\widetilde{\tau}, \widetilde{\eta}], * F(A)\right)
$$

vanishes for vectors $\widetilde{\tau}, \widetilde{\eta} \in T_{A} \mathcal{A}^{*}$ tangent to the gauge fibers $\widetilde{\tau}=D_{A} \phi$. However, $\widetilde{\alpha}_{k}$ is trivial only if the holonomy group associated to any closed curve contained in a gauge orbit is trivial. This is only possible if the projection $\Omega_{k}$ of the curvature 2 -form $\widetilde{\Omega}_{k}$ to the space of irreducible gauge orbits $\mathcal{M}^{*}=\mathcal{A}^{*} / \mathcal{G}$,

$$
\Omega_{k}(\tau, \eta)=\widetilde{\Omega}_{k}\left(\widetilde{\tau}^{h}, \widetilde{\eta}^{h}\right)
$$

belongs (modulo a factor $2 \pi$ ) to an integer cohomology class of $\mathcal{M}^{*}$, i.e.

$$
\frac{1}{2 \pi}\left[\Omega_{k}\right] \in H^{2}\left(\mathcal{M}^{*}, \mathbb{Z}\right) .
$$

In equation (5.5) $\widetilde{\tau}^{h}$ denotes the horizontal component of any tangent vector $\widetilde{\tau} \in$ $T_{A} \mathcal{A}^{*}$ with projection $\tau \in T_{[A]} \mathcal{M}^{*}$ which is orthogonal to the gauge fiber at $A$ with respect to the canonical $L^{2}$ product defined in $\mathcal{A}^{*}$ by the Riemaniann metric of $\Sigma$, i.e. $\widetilde{\tau}^{h}=P_{A} \widetilde{\tau}, P_{A}=\left(I-D_{A} G_{A} D_{A}^{*}\right)$ being the corresponding orthogonal projector.

The condition (5.6) is satisfied if and only if the Chern-Simons charge $k$ is an integer. Because of the triviality of $\widetilde{\alpha}_{k}$ when $k$ is an integer, the action of the group of gauge transformations $\mathcal{G}$ can be globally lifted to an action on the line bundle $\mathcal{A}^{*} \times \mathbb{C}$. Gauss law implies the invariance of quantum states under this action. Thus, the quantum states can be completely characterized by sections of the line bundle $\mathcal{E}_{k}\left(\mathcal{M}^{*}, \mathbb{C}\right)$ defined by the gauge orbits $\mathcal{E}_{k}=\mathcal{A}^{*} \times \mathbb{C} / \mathcal{G}$ of such an action. It is obvious that the first Chern class of this bundle $c_{1}\left(\mathcal{E}_{k}\right)=\frac{1}{2 \pi}\left[\Omega_{k}\right]$ is non-trivial provided $k \neq 0$

In the same way, the connection $\widetilde{\alpha}_{k}$ of $\mathcal{A}^{*} \times \mathbb{C}$ projects down to a connection $\alpha_{k}$ in $\mathcal{E}_{k}$ and the quantum hamiltonian can be expressed as an operator

$$
\mathbb{H}=-\frac{g^{2}}{2}\left\|\nabla_{\alpha_{k}}\right\|^{2}+\frac{1}{2 g^{2}}\|F(A)\|^{2}+\frac{k^{2} g^{2}}{8 \pi^{2}}\left(* F(A), G_{A} * F(A)\right)
$$

acting on the sections of $\mathcal{E}_{k}$. 
THEOREM 5.3. Every physical state has nodal configurations on the orbit space of irreducible connections $\mathcal{M}^{*}$

Proof. Since the Chern class of the line bundle $\mathcal{E}_{k}\left(\mathcal{M}^{*}, \mathbb{C}\right)$ does not vanish $c_{1}\left(\mathcal{E}_{k}\right)=\left[\Omega_{k}\right] / 2 \pi \neq 0$, the bundle $\mathcal{E}_{k}\left(\mathcal{M}^{*}, \mathbb{C}\right)$ is non-trivial and cannot have sections (i.e. physical states) without vanishing points over $\mathcal{M}^{*}[\mathbf{1}]$.

We have seen that physical states present two kind of nodes: those located at reducible connections with unstable bundles (Proposition 5.1) and the new ones required by Theorem 5.3 to appear at irreducible connections. The first type of nodes can be thought as kinematical nodes, they appear for any physical state at the same configurations because of its geometrical origen based on the global properties of Gauss law.

However, the second type of nodes whose existence is inferred from theorem 5.3 are genuine non-abelian configurations and might depend on the physical states we consider. To find these irreducible nodal configurations a more elaborate dynamical argument is required. In general, these nodal configurations will not be the same for all physical states. For such a reason they can be considered as dynamical nodes.

It has been conjectured [3] that the nodes of the vacuum state $\Psi_{0}$, i.e. the eigenstate of the hamiltonian (5.1) with lowest eigenvalue, are located at connections which are associated to unstable bundles. The conjecture is based on the Ritz variational argument which establishes that the expectation value of the hamiltonian $\mathbb{H}$ on the space of quantum physical states must attaint its minimum value for the ground state $\Psi_{0}$. It is trivial to see that the lowest eigenstates of the kinetic term of the hamiltonian (5.1) are the Chern-Simons states discussed on the previous section $[\mathbf{2}]$ and they vanish for the strata associated unstable bundles. The contribution of the potential term attaints its minimal value on these strata at the reducible connections which satisfy the Yang-Mills equation according to the Atiyah-Bott result [8]. But the vacuum state as any other physical state must vanish for such connections. The vanishing of the vacuum wave functional $\Psi_{0}(A)$ along the whole strata seems, thus, necessary to minimize the expectation value of the hamiltonian $\mathbb{H}$. This behavior is not only required for the minimization of the kinetic term, but also for that of the Yang-Mills potential term. Both terms, kinetic and potential, of the hamiltonian conspire to force the vanishing of the vacuum on connections associated to unstable bundles [3].

REMARK . Such a behavior is in contrast with Feynman's claim on the absence of nodes in the ground state of pure Yang-Mills theory in 2+1 dimensions [12]. Another difference between both theories is that in Yang-Mills theory fermions are confined whereas in the topologically massive theory they are not. This relationship between the absence of confinement and the existence of nodes in the vacuum state, suggests that those configurations where the vacuum vanishes might play a relevant role in the mechanism of confinement.

In compact lattice $\mathrm{QED}_{2+1}$ it has been shown that the logarithmic perturbative Coulomb potential becomes linear by means of Debye screening of electric charges in a monopole gas $[\mathbf{2 7}]$ in a similar manner as vortices drive the BerezinskiiKosterlitz-Thouless phase transition in the XY model [9] [19]. The above conjecture indicates that connections associated to unstable bundles would play a similar role in the mechanism of quark confinement of the non-abelian theory. This provides a 
geometric setting for the 't Hooft-Mandelstam scenario [18][22] in 2+1 dimensional gauge theories.

REMARK . Although properly speaking, there are not unstable bundles for infinite volumes $\left(\Sigma=\mathbb{R}^{2}\right)$, it is possible to distinguish holomorphic bundles which can be extended at infinity to stable bundles defined over the sphere from the ones which do not admit such an extension. Actually, the bundles of the second class persist as nodes of the quantum vacuum which suggests that their contribution to the confinement mechanism survives to the infinite volume limit

\section{Four-dimensional Gauge Theories.}

The prominent relevance of unstable bundles in $2+1$ dimensional theories raises the question about their possible implications in $3+1$ dimensional gauge theories.

Among the interesting classical configurations which have been conjectured to have a role in non-perturbative effects in four dimensions there are two leading configurations: instantons and sphalerons. Both solutions appear as classical solutions of Yang-Mills equations.

Instantons are solutions of the selfdual equations and therefore they are strict minima of the Euclidean Yang-Mills functional in the corresponding non-trivial bundle. Instantons with unit topological charge and structure group $S U(2)$ are given by

$$
A_{\mu}=\frac{2 \tau_{\mu \nu}\left(x-x_{0}\right)^{\nu}}{\left(x-x_{0}\right)^{2}+\rho^{2}}
$$

in stereographic coordinates of the four dimensional sphere $S^{4}$. There are two collective coordinates which parametrize the moduli space of $k=1 S U(2)$ instantons: the radius $\rho$ and its center $x_{0}$. The $\mathfrak{s l}(2, \mathbb{C})$ matrices $\tau_{\mu \nu}=i\left(\tau_{\nu}^{\dagger} \tau_{\mu}-\delta_{\mu \nu}\right)$, with $\tau=(-I, i \vec{\sigma})$ define a coupling between internal and external degrees of freedom.

Although the role of instantons as main responsible of the non-perturbative contributions associated to tunnel effect between classical vacua seems to be very similar to that of monopoles in compact QED in three-dimensional space-times, their contribution to confinement does not seem to be crucial. Indeed, a standard argument due to Witten shows that their contribution is exponentially suppressed in the large $\mathrm{N}$ limit, whereas quark confinement is strengthened in that limit [30]. However, the instanton contribution is very relevant for the problem of chiral symmetry breaking in the presence of dynamical quarks [10].

Sphalerons are static solutions of Yang-Mills equations which can only exist for finite space volumes. They are unstable and become characterized by the existence of a finite number of unstable decaying modes. The value of the Yang-Mill functional on them marks the height of the potential barrier between classical vacua and, therefore, is related to the transition temperature necessary for the appearance of direct coalescence between those vacua. In $2+1$ dimensional gauge theories Atiyah and Bott showed that in any strata of $\mathcal{A}$ there exist sphalerons [8]. They correspond to the magnetic monopoles described in the previous section, which where identified as kinematic nodes of all physical states in topological massive gauge theory. In 3+1 dimensions their existence was predicted by Manton [23] by an argument based on the non-simply connected nature of the orbit space of 3 dimensional gauge fields and a generalization of the Ljusternik-Snierelman theory 
[21]. Explicit expression for some sphalerons on $S^{3}$ can be obtained from the observation that the pullback of the instanton to a 3 -dimensional sphere embedded on $S^{4}$ with origin on the center of the instanton and the same radius that the instanton is an unstable critical point of the 3-dimensional Yang-Mills functional. For $S U(2)$ the sphaleron in stereographic coordinates reads

$$
A_{j}=\frac{4 \rho}{\left(x^{2}+4 \rho^{2}\right)^{2}}\left(4 \rho \epsilon_{j k}^{a} x^{k}-2 x^{a} x_{j}+\left[x^{2}-4 \rho^{2}\right] \delta_{j}^{a}\right) \sigma_{a} .
$$

The unstable mode can be identified with the variation under scale transformations.

The role of sphalerons in the quantum theory of gauge fields comes from the following result.

TheOREM 6.1. The vacuum state of the quantum Yang-Mills theory with a $\theta$-term ( $\theta$-vacuum) has a node at any classical sphaleron configuration for $\theta=\pi$.

See [4] for a proof.

Since the theory is expected to deconfine for such a value of the theta parameter, the result suggest that those nodes might be responsible for the confining properties of the vacuum in absence of $\theta$ term where the vacuum has no classical nodal configurations [12].

What is striking is that both types of connections, instantons and sphalerons, are related to unstable bundles. If we consider the restriction of the sphaleron (6.2) to a two dimensional sphere $S^{2}$ centered at $(0,0, \mathrm{c})$ and radius $a$ we obtain in stereographic coordinates the following two-dimensional connection

$$
A_{\bar{z}}=\frac{\gamma}{\left(1+|z|^{2}\right)^{2}}\left(\begin{array}{cc}
-z & -1 \\
z^{2} & z
\end{array}\right)
$$

with $\gamma=2 a(a-i c) /\left(a^{2}+\rho^{2}+c^{2}\right)$. It is easy to show that such a connection corresponds to semi-stable bundle for all values of $\gamma$, except for $\gamma=1$ where as shown Example 2.2 it corresponds to an unstable bundle. Such an especial configuration emerges when the radius of the embedded $S^{2}$ sphere equals that of the sphaleron $a=\rho$ and is centered at the origin $c=0$. This establishes a clear connection between sphalerons and unstable bundles.

In the same way it can be shown that instantons have a correspondence with unstable bundles. If we considered the embedding of a 2-dimensional sphere $S^{2}$ of radius $a$ on $\mathbb{R}^{4}$ with origin at $x=(c, 0,0,0)$. The pullback of the instanton centered at $x=(0,0,0,0)$ to $S^{2}$ by such an embedding is again given by $(6.3)$ with

$$
\gamma=\frac{2 a(a-i c)}{a^{2}+\rho^{2}+c^{2}}
$$

Once more when and only $c=0$ and $a=\rho$ we get an unstable bundle, the same one induced by the sphaleron.

This relation between instantons and unstable bundles can be generalized for instantons with higher topological charges. The 2 -instanton configuration with two symmetric centers at $x_{+}=\left(x_{0}, 0,0,0\right)$ and $x_{-}=\left(-x_{0}, 0,0,0\right)$ and one single scale $\rho$ reads,

with

$$
A_{\mu}=2 \bar{\tau}_{\mu \nu} \partial^{\nu} \phi(x)
$$

$$
\phi(x)=\log \left(1+\frac{\rho^{2}}{\left(x-x_{+}\right)^{2}}+\frac{\rho^{2}}{\left(x-x_{-}\right)^{2}}\right)
$$


and $\bar{\tau}_{\mu \nu}=i\left(\bar{\tau}_{\nu}^{\dagger} \bar{\tau}_{\mu}-\delta_{\mu \nu}\right), \bar{\tau}=(-I,-i \vec{\sigma})$. The corresponding pullback to a two dimensional sphere centered at $x=(c, 0,0,0)$ with radius $a$ is again of the form (6.3) with

$$
\gamma=-\frac{a}{\rho^{2}} \frac{2 i}{1+\alpha_{+}+\alpha_{-}}\left[\alpha_{+}^{2}\left(c+x_{0}+i a\right)+\alpha_{-}^{2}\left(c-x_{0}+i a\right)\right]
$$

with $\left.\alpha_{ \pm}=\rho^{2} /\left[x_{0} \pm c\right)^{2}+a^{2}\right]$. Therefore, in this case we have two different spheres with unstable bundles. If the distance between the two instanton centers is larger than the instanton size, $\rho<2 x_{0}$, the two spheres have the same radius $a \in\left(0, \sqrt{3} x_{0}\right)$ and are centered at two symmetric points $c_{ \pm}=\left( \pm c\left(\rho, x_{0}\right), 0,0,0\right)$ with $c\left(\rho, x_{0}\right) \in$ $\left(0, x_{0}\right)$. When the size of the instanton $\rho \downarrow 0$ the radius of the spheres $a \downarrow 0$ and the centers go to $c_{ \pm} \rightarrow x_{ \pm}$. If the two instantons are far apart $x_{0} \uparrow \infty$ the centers of the spheres go to $x_{ \pm}$with radius $a=\rho$, as should correspond to two isolated instantons. For instantons of larger radius $\rho>2 x_{0}$ the two spheres are centered on the origin with radii

$$
a_{ \pm}=\sqrt{\rho^{2}-x_{0}^{2} \pm \rho \sqrt{\rho^{2}-4 x_{0}^{2}}}
$$

In the limit $x_{0} \downarrow 0$ when the distance between the instantons becomes negligible $a_{+} \rightarrow \sqrt{2} \rho$ which agrees with the fact that the two instantons merge into a single instanton with radius $\sqrt{2} \rho$.

The same connection with unstable bundles appears for higher order multiinstantons. This is not surprising because there are topological reasons which imply that generic multi-instanton connections must induce unstable bundles in some 2dimensional spheres.

Proposition 6.2. A generic unitary connection $A$ on a principal bundle $P\left(S^{4}, S U(N)\right)$ with non-trivial second Chern class $c_{2}(A) \neq 0$ induces an unstable bundle over some 2-dimensional sphere $S^{2}$ embedded in $S^{4}$.

Proof. It is a straighforward generalization of the Asorey-Mitter result proved in Ref. [6] for connections over $S^{2} \times S^{2}$. A generic unitary connection $A$ on a principal bundle $P\left(S^{2} \times S^{2}, S U(N)\right)$ with non-trivial second Chern class $c_{2}(A)=k$ induces by dimensional reduction a non trivial 2-dimensional cycle $\sigma$ on the orbit space of connections of the trivial bundle $S^{2} \times S U(N)$. In fact $\sigma$ belongs to the $k$ class of second homotopy group of $\mathcal{M}, \pi_{2}(\mathcal{M})=\mathbb{Z}[\mathbf{6}]$. The orbits of the connections induced by dimensional reduction are essentially defined by the pullbacks of $A$ to a family of 2-dimensional spheres $S^{2}$ embedded in $S^{4}$ (see Ref. [6] for details). The generalization of the result for connections over the sphere $S^{4}$ is straightforward.

Since the space of gauge orbits $\mathcal{M}_{\text {ss }}$ induced from semi-stable bundles of $S^{2} \times$ $S U(N)$ has a trivial cohomology group $\pi_{2}\left(\mathcal{M}_{\mathrm{ss}}\right)=0$ any non-trivial cycle $\sigma$ on $\pi_{2}\left(\mathcal{M}^{*}\right)=\mathbb{Z}$ must cross some of the orbits associated to unstable bundles.

However, the connection with unstable bundles is not exclusive of 4-dimensional gauge fields with non-vanishing $c_{2}$ Chern class. It is trivial to find examples of connections belonging the classical vacuum sector with null Chern class, $c_{2}(A)=0$, which also induce unstable bundles on some 2-dimensional spheres. 


\section{Conclusions}

We have analyzed the structure of connections which appear as nodes of vacuum states in some quantum field theories. A common characteristic of all those connections is their relation with unstable bundles. Since in the corresponding quantum theories the quark interaction is shown to be non-confining, it has been conjectured that there must exist a relation between those configurations and the mechanism of quark confinement in gauge theories where such a nodes do not appear. On the other hand the fact that all those connections are in a way or another related with unstable bundles suggests that it precisely this feature which is on the root of such a mechanism. To some extent the association of connections with unstable bundles on 2-dimensional embedded surfaces can be considered as an intrinsic generalization of the concept of magnetic monopoles for non-abelian gauge theories. From this perspective the above conjecture about the role of those configurations on the confinement problem can be considered as a generalization of 't Hooft-Mandelstam dual superconductor conjecture.

\section{References}

1. M. Asorey, Topological Effects in Yang-Mills Theory in 2+1 Dimensions, Geometry and Fields (A. Jadczyk, ed.), World Scientific, Singapore, 1986.

2. M. Asorey, Topological Phases of Quantum Theories. Chern-Simons Theory, J. Geom. Phys. 11 (1993), 63-94.

3. M. Asorey, F. Falceto, J. L. López, G. Luzón, Nodes Monopoles and Confinement in 2+1 Dimensional Gauge Theories, Phys. Lett. B349 (1995), 125-130.

4. M. Asorey, F. Falceto, Vacuum Nodes in $Q C D$ at $\theta=\pi$ : Exact Results, Phys. Rev. Lett. 77 (1996), 3074-3077.

5. M. Asorey, P.K. Mitter, Cohomology of the Gauge Orbit Space and 2+1 Dimensional YangMills Theory with Chern-Simons Term, Phys. Lett. B153 (1985), 147-152.

6. M. Asorey, P.K. Mitter, Cohomology of the Yang-Mills Gauge Orbit Space and Dimensional Reduction, Ann. Inst. Poincaré 45 (1986), 61-87.

7. M. Atiyah, Vector bundles over an elliptic curve, Proc. London Math. Soc. 7 (1957), 414-452.

8. M. Atiyah, R. Bott, The Yang-Mills Equations over Riemann Surfaces, Philos. Trans. Roy. Soc. London A308 (1982), 523-615.

9. V.L. Berezinskii, Destruction of Long-Range Order in One-Dimensional and Two-Dimensional Systems Having a Continuous Symmetry Group I: Classical Systems, Sov. Phys. JETP 32 (1971), 493-500.

10. D. Diakonov, V. Petrov, Chiral condensate in the instanton vacuum, Phys. Lett. 147B (1984), 351-356; Sov. Phys. JETP 62 (1985), 204-214, 431-437; A theory of light quarks in the instanton vacuum, Nucl. Phys. B272 (1986), 457-489.

11. F. Falceto, K. Gawedzki, Chern-Simons States at Genus One, Commun. Math. Phys. 159 (1994), 549-579.

12. R. Feynman, The Qualitative Behavior of Yang-Mills Theory in 2+1-Dimensions, Nucl. Phys. B188 (1981), 479-512.

13. R. Friedman, J. W. Morgan, E. Witten, Vector bundles and F-theory, Commun. Math. Phys. 187 (1997), 679-743.

14. R. Friedman, J. W. Morgan, E. Witten, Principal G-bundles over elliptic curves, hep-th/ 9707004 (1997).

15. K. Gawedzki, A. Kupiainen, SU(2) Chern-Simons Theory at Genus Zero, Commun. Math. Phys. 135 (1991), 531-546.

16. A. Grothendieck, Sur la classification des fibrés holomorphes sur la sphère de Riemann, Amer. J. Math. 79 (1956), 121-138.

17. R.C. Gunning, Lectures on Vector Bundles over Riemann Surfaces, Princeton University Press, Princeton NJ, 1967. 
18. 't Hooft, Gauge Theories with Unified Weak Electromagnetic and Strong Interactions, High Energy Physics (A. Zichichi, eds.), Compositori, Bologna, 1976; Topology of the Gauge Condition and new Confinement Phases in non-Abelian Gauge Theories, Nucl. Phys. B190 (1981), 455-478.

19. J.M. Kosterlitz, D.J. Thouless, Ordering, metastability and phase transitions in two-dimensional systems, J. Phys. C6 (1973), 1181-1203.

20. A. Kupiainen, J. Mickelsson, What is the Effective Action in Two Dimensions?, Phys. Lett. B185 (1987), 107-110.

21. L. A. Ljusternik, L. Schnierelman, Methodes topologiques dans les problèmes variationels, Gauthier-Villars, Paris, 1934;L. A. Ljusternik, Topology of the calculus of variations in the large, Amer. Math. Soc. Tranl. 16 (1966), Amer. Math. Soc., Providence, RI.

22. S. Mandelstam, Vortices and Quark Confinement in non-Abelian Gauge Theories, Phys. Rep. 23 (1976), 245-249; General Introduction to Confinement, Phys. Rep. 67 (1980), 109-121.

23. N. Manton, Topology in The Weinberg-Salam Theory, Phys. Rev. D28 (1983), 2019-2026;F.R. Klinkhamer, N. Manton, A Saddle Point Solution In The Weinberg-Salam Theory, Phys. Rev. D30 (1984), 2212-2220.

24. D. Mumford, Geometric invariant theory, Springer-Verlag, Berlin, 1965; Vector bundles over an elliptic curve, Proc. Int. Congress of Math., vol. Stockholm, 1962, pp. 526-530.

25. M.S. Narasimhan and S. Ramanan, Deformations of the moduli space of vector bundles over an algebraic curve, Ann. of Math. 101 (1975), 39-47.

26. M.S. Narasimhan and C. S. Seshadri, Stable and unitary bundles on a compact Riemann surface, Ann. of Math. 82 (1965), 540-567.

27. A. Polyakov, Compact Gauge Fields and the Infrared Catastrophe, Phys. Lett. B59 (1975), 82-84; Quark Confinement and Topology of Gauge Groups, Nucl. Phys. 120 (1977), 429-458.

28. S. Ramanan, The moduli spaces of vector bundles over an algebraic curve, Math. Ann. 101 (1973), 69-84.

29. Ward, On self-dual gauge fields, Phys. Lett. A61 (1977), 81-82.

30. E. Witten, Instantons, the Quark Model, and the 1/N Expansion, Nucl. Phys. B149 (1979), 285-320.

$\dagger$ Departamento de Física Teórica, Facultad de Ciencias, Universidad de Zaragoza, 50009 Zaragoza, Spain

$\ddagger$ Department of Physics, Theoretical Physics, University of Oxford, 1 Keble RoAd, OXford, OX1 3NP United Kingdom 